\title{
ON THE SUM OF SQUARES OF MIDDLE-THIRD CANTOR SET
}

\author{
ZHIQIANG WANG, KAN JIANG, WENXIA LI, AND BING ZHAO
}

\begin{abstract}
Let $C$ be the middle-third Cantor set. In this paper, we show that for every $x \in[0,4]$, there exist $x_{1}, x_{2}, x_{3}, x_{4} \in C$ such that $x=x_{1}^{2}+x_{2}^{2}+x_{3}^{2}+x_{4}^{2}$, which was conjectured in [Athreya, J. S.; Reznick, B.; Tyson, J. T. Cantor set arithmetic. Amer. Math. Monthly 126 (2019), no. 1, 4-17].
\end{abstract}

\section{INTRODUCTION}

The middle-third Cantor set

$$
C=\left\{\sum_{i=1}^{\infty} \frac{\varepsilon_{i}}{3^{i}}: \varepsilon_{i} \in\{0,2\}\right\}
$$

is a classical object in fractal geometry. The arithmetic on middle-third Cantor set has been studied in [1, 2, 3, 4, 5, 6, 8]. The first classical result is that the set

$$
C-C:=\{x-y: x, y \in C\}
$$

equals to the interval $[-1,1]$. The proof of (1.1) was first given by H. Steinhaus in 1917. The result was rediscovered by J. F. Randolph in 1940 [7]. Using the symmetry of $C$, we can deduce that

$$
C+C=C+(1-C)=1+(C-C)=[0,2],
$$

where $C+C:=\{x+y: x, y \in C\}$. The multiplication and division on middle-third Cantor set were discussed in [1]. They proved that

$$
\mathcal{L}(C \cdot C) \geq \frac{17}{21} \text { and } \frac{C}{C}=\bigcup_{n=-\infty}^{\infty}\left[\frac{2}{3} \cdot 3^{n}, \frac{3}{2} \cdot 3^{n}\right] \cup\{0\},
$$

where $C \cdot C:=\{x y: x, y \in C\}, \frac{C}{C}:=\left\{\frac{x}{y}: x, y \in C, y \neq 0\right\}$ and $\mathcal{L}$ denotes the Lebesgue measure on $\mathbb{R}$. Gu, Jiang, $\mathrm{Xi}$ and Zhao 44 gave the complete topological structure of $C \cdot C$. Moreover, they also proved that the Lebesgue measure of $C \cdot C$ is about 0.80955 .

The main motivation of this paper is due to a conjecture posed by Athreya, Reznick and Tyson [1]. They conjectured $\left\{x_{1}^{2}+x_{2}^{2}+x_{3}^{2}+x_{4}^{2}: x_{i} \in C\right\}=[0,4]$ and claimed that there is strong numerical evidence supporting it. In this paper, we will prove this conjecture.

Fixing $\alpha>1$, let $C_{\alpha}$ (the middle- $\frac{1}{\alpha}$ Cantor set) be generated by the iterated function system $\Phi=\left\{f_{1}(x)=r x, f_{2}(x)=r x+1-r\right\}$ with $r=\frac{1}{2}\left(1-\frac{1}{\alpha}\right)$. Thus the classical middlethird Cantor set $C=C_{3}$. In the present paper we prove

Date: January 15, 2020.

2010 Mathematics Subject Classification. Primary: 28A80, Secondary:11K55.

Key words and phrases. middle-third Cantor set, middle- $\frac{1}{\alpha}$ Cantor set, sum of four squares. 
Theorem 1.1. Let $C_{\alpha}$ be the middle- $\frac{1}{\alpha}$ Cantor set for $\alpha>1$. Then

$$
\left\{x_{1}^{2}+x_{2}^{2}+x_{3}^{2}+x_{4}^{2}: x_{i} \in C_{\alpha}\right\}=[0,4] \text { if and only if } \alpha \geq 3 \text {. }
$$

This paper is organized as follows. In section 2 , we discuss the set $\left\{x_{1}^{2}+x_{2}^{2}+x_{3}^{2}: x_{i} \in C_{\alpha}\right\}$. The proof of Theorem 1.1 is arranged in the section 3 .

\section{Sum OF THREE SQUARES}

As stated in the previous section, $C_{\alpha}$ is the unique nonempty compact set satisfying

$$
C_{\alpha}=f_{1}\left(C_{\alpha}\right) \cup f_{2}\left(C_{\alpha}\right)=r C_{\alpha} \cup\left(r C_{\alpha}+1-r\right)
$$

where $r=\frac{1}{2}\left(1-\frac{1}{\alpha}\right)$. It follows that if $x \in C_{\alpha}$, then $r x \in C_{\alpha}$. We will use this simple observation in Lemma 3.1. For each positive integer $n$ let

$$
\mathcal{F}_{n}=\left\{f_{\sigma}([0,1]): \sigma \in\{1,2\}^{n}\right\} \text { and } F_{n}=\bigcup_{A \in \mathcal{F}_{n}} A,
$$

where $f_{\sigma}(x)=f_{\sigma_{1}} \circ f_{\sigma_{2}} \circ \cdots \circ f_{\sigma_{n}}(x)$ for $\sigma=\sigma_{1} \sigma_{2} \cdots \sigma_{n} \in\{1,2\}^{n}$. Then the sequence $F_{n}, n=1,2, \cdots$, of nonempty compact sets is decreasing and

$$
C_{\alpha}=\bigcap_{n=1}^{\infty} F_{n}=\bigcap_{n=1}^{\infty} \bigcup_{\sigma \in\{1,2\}^{n}} f_{\sigma}([0,1]) .
$$

It is easy to see that for $\sigma=\sigma_{1} \sigma_{2} \cdots \sigma_{n} \in\{1,2\}^{n}$

$$
f_{\sigma}(0)=\frac{1-r}{r} \sum_{k=1}^{n}\left(\sigma_{k}-1\right) r^{k}
$$

and so

$$
f_{\sigma}([0,1])=\left[f_{\sigma}(0), f_{\sigma}(1)\right]=\left[\frac{1-r}{r} \sum_{k=1}^{n}\left(\sigma_{k}-1\right) r^{k}, \frac{1-r}{r} \sum_{k=1}^{n}\left(\sigma_{k}-1\right) r^{k}+r^{n}\right] .
$$

Each element of $\mathcal{F}_{n}$, called an $n$-level basic interval, has length $r^{n}$. For an $n$-level basic interval $f_{\sigma}([0,1])$, it contains two $(n+1)$-level basic intervals $f_{\sigma 1}([0,1])$ and $f_{\sigma 2}([0,1])$. $f_{\sigma}([0,1])$ shares the same left endpoint with $f_{\sigma 1}([0,1])$, and shares the same right endpoint with $f_{\sigma 2}([0,1])$. The length of the open interval $f_{\sigma}([0,1]) \backslash\left(f_{\sigma 1}([0,1]) \cup f_{\sigma 2}([0,1])\right)$ is $\frac{1}{\alpha}$ times that of $f_{\sigma}([0,1])$.

Denote by $L_{n}$ the collection of left endpoints of all $n$-level basic intervals. For $u \in L_{n}$, we associate $u$ with an $n$-level basic interval

$$
I_{u}=\left[u, u+r^{n}\right]
$$

and two $(n+1)$-level basic intervals denoted by

$$
I_{u, 0}=\left[u, u+r^{n+1}\right], I_{u, 1}=\left[u+(1-r) r^{n}, u+r^{n}\right] .
$$

The key to discuss the sum of squares of Cantor set is the following lemma, which is an easy exercise in real analysis. 
Lemma 2.1. Let $\varphi: \mathbb{R}^{d} \rightarrow \mathbb{R}$ be continuous. If $\left\{K_{j}\right\}_{j \in \mathbb{N}}$ is a decreasing sequence of nonempty compact subsets of $\mathbb{R}^{d}$, then

$$
\varphi\left(\bigcap_{j=1}^{\infty} K_{j}\right)=\bigcap_{j=1}^{\infty} \varphi\left(K_{j}\right) .
$$

Proof. Since $\bigcap_{j=1}^{\infty} K_{j} \subseteq K_{n}$ for every $n \in \mathbb{N}$, we have $\varphi\left(\bigcap_{j=1}^{\infty} K_{j}\right) \subseteq \bigcap_{j=1}^{\infty} \varphi\left(K_{j}\right)$. Conversely, assume that $y \in \bigcap_{j=1}^{\infty} \varphi\left(K_{j}\right)$. For every $j$, we can find $x_{j} \in K_{j}$ such that $\varphi\left(x_{j}\right)=y$. Since $K_{1}$ is compact, by Bolzano-Weierstrass Theorem, there is a convergent subsequence $x_{n_{j}} \rightarrow x$. Since $\varphi$ is continuous, we have $\varphi(x)=y$. Note that the sequence $\left\{x_{n_{j}}\right\}_{j \geq m}$ is in $K_{m}$ for every $m \in \mathbb{N}$. It follows from compactness that $x \in K_{m}$ for every $m \in \mathbb{N}$. Therefore, $y=\varphi(x) \in \varphi\left(\bigcap_{j=1}^{\infty} K_{j}\right)$, which completes the proof.

Define functions $g: \mathbb{R}^{3} \rightarrow \mathbb{R}$ and $f: \mathbb{R}^{4} \rightarrow \mathbb{R}$ by letting

$$
g\left(x_{1}, x_{2}, x_{3}\right)=x_{1}^{2}+x_{2}^{2}+x_{3}^{2}
$$

and

$$
f\left(x_{1}, x_{2}, x_{3}, x_{4}\right)=g\left(x_{1}, x_{2}, x_{3}\right)+x_{4}^{2}=x_{1}^{2}+x_{2}^{2}+x_{3}^{2}+x_{4}^{2} .
$$

For a positive integer $k$ and a nonempty set $A \subseteq \mathbb{R}$, denote $A^{k}=\left\{\left(x_{1}, \cdots, x_{k}\right): x_{i} \in A\right\}$. In order to show $f\left(C_{\alpha}^{4}\right)=[0,4]$, we need to discuss the set $g\left(C_{\alpha}^{3}\right)$ and find some intervals in $g\left(C_{\alpha}^{3}\right)$. Note that $C_{\alpha}^{3}=\bigcap_{n=1}^{\infty} F_{n}^{3}$. Applying Lemma 2.1 for the continuous function $g$, we obtain the following corollary.

Corollary 2.2. $g\left(C_{\alpha}^{3}\right)=\bigcap_{n=1}^{\infty} g\left(F_{n}^{3}\right)$.

If an interval $I \subseteq g\left(F_{n}^{3}\right)$ for every $n \in \mathbb{N}$, then $I \subseteq g\left(C_{\alpha}^{3}\right)$. The following two lemmas give a sufficient condition to find intervals in $g\left(C_{\alpha}^{3}\right)$.

Lemma 2.3. Let $\alpha \geq 3$. For any $u, v, w \in L_{n}$, if

$$
\max \{u, v, w\}>0
$$

and

$$
4(1-r) \max \{u, v, w\} \leq 2(u+v+w)+(1+2 r) r^{n},
$$

then

$$
g\left(I_{u} \times I_{v} \times I_{w}\right)=g\left(\left(I_{u, 0} \cup I_{u, 1}\right) \times\left(I_{v, 0} \cup I_{v, 1}\right) \times\left(I_{w, 0} \cup I_{w, 1}\right)\right) .
$$

Proof. At first we have $r=\frac{1}{2}\left(1-\frac{1}{\alpha}\right) \in[1 / 3,1 / 2)$ since $\alpha \geq 3$. Write $t=u^{2}+v^{2}+w^{2}$. Without loss of generality, we can assume that $u \geq v \geq w$. By (2.1) we have $u>0$ and so $u \geq f_{1^{n-1}{ }_{2}}(0)=(1-r) r^{n-1}>r^{n}$. In addition, (2.2) reduces to

$$
2 v+2 w+(1+2 r) r^{n} \geq 2(1-2 r) u .
$$

It is routine to verify that

$$
\begin{gathered}
g\left(I_{u, 1} \times I_{v, 0} \times I_{w, 0}\right)=\left[t+2 u(1-r) r^{n}+(1-r)^{2} r^{2 n}, t+2(u+r v+r w) r^{n}+\left(1+2 r^{2}\right) r^{2 n}\right], \\
g\left(I_{u, 1} \times I_{v, 0} \times I_{w, 1}\right)=\left[t+2(u+w)(1-r) r^{n}+2(1-r)^{2} r^{2 n}, t+2(u+r v+w) r^{n}+\left(2+r^{2}\right) r^{2 n}\right], \\
g\left(I_{u, 1} \times I_{v, 1} \times I_{w, 0}\right)=\left[t+2(u+v)(1-r) r^{n}+2(1-r)^{2} r^{2 n}, t+2(u+v+r w) r^{n}+\left(2+r^{2}\right) r^{2 n}\right],
\end{gathered}
$$


and

$$
g\left(I_{u, 1} \times I_{v, 1} \times I_{w, 1}\right)=\left[t+2(u+v+w)(1-r) r^{n}+3(1-r)^{2} r^{2 n}, t+2(u+v+w) r^{n}+3 r^{2 n}\right] .
$$

Note that

$$
\begin{aligned}
& t+2(u+r v+r w) r^{n}+\left(1+2 r^{2}\right) r^{2 n}-\left(t+2(u+w)(1-r) r^{n}+2(1-r)^{2} r^{2 n}\right) \\
= & 2(r u+r v+2 r w-w) r^{n}+(4 r-1) r^{2 n} \\
\geq & 2(4 r-1) w r^{n}+(4 r-1) r^{2 n}>0,
\end{aligned}
$$

and

$$
\begin{aligned}
& t+2(u+r v+w) r^{n}+\left(2+r^{2}\right) r^{2 n}-\left(t+2(u+v)(1-r) r^{n}+2(1-r)^{2} r^{2 n}\right) \\
= & 2(r u+2 r v-v+w) r^{n}+(4-r) r^{2 n+1} \\
\geq & 2(3 r-1) v r^{n}+(4-r) r^{2 n+1}>0,
\end{aligned}
$$

and

$$
\begin{aligned}
& t+2(u+v+r w) r^{n}+\left(2+r^{2}\right) r^{2 n}-\left(t+2(u+v+w)(1-r) r^{n}+3(1-r)^{2} r^{2 n}\right) \\
= & 2(r u+r v+2 r w-w) r^{n}+\left(6 r-2 r^{2}-1\right) r^{2 n} \\
\geq & 2(4 r-1) w r^{n}+\left(6 r-2 r^{2}-1\right) r^{2 n}>0 .
\end{aligned}
$$

Therefore, we have

$$
\begin{aligned}
& g\left(I_{u, 1} \times\left(I_{v, 0} \cup I_{v, 1}\right) \times\left(I_{w, 0} \cup I_{w, 1}\right)\right) \\
= & {\left[t+2 u(1-r) r^{n}+(1-r)^{2} r^{2 n}, t+2(u+v+w) r^{n}+3 r^{2 n}\right] . }
\end{aligned}
$$

It is also routine to verify that

$$
\begin{gathered}
g\left(I_{u, 0} \times I_{v, 0} \times I_{w, 0}\right)=\left[t, t+2(u+v+w) r^{n+1}+3 r^{2 n+2}\right], \\
g\left(I_{u, 0} \times I_{v, 0} \times I_{w, 1}\right)=\left[t+2 w(1-r) r^{n}+(1-r)^{2} r^{2 n}, t+2(r u+r v+w) r^{n}+\left(1+2 r^{2}\right) r^{2 n}\right], \\
g\left(I_{u, 0} \times I_{v, 1} \times I_{w, 0}\right)=\left[t+2 v(1-r) r^{n}+(1-r)^{2} r^{2 n}, t+2(r u+v+r w) r^{n}+\left(1+2 r^{2}\right) r^{2 n}\right],
\end{gathered}
$$

and

$$
g\left(I_{u, 0} \times I_{v, 1} \times I_{w, 1}\right)=\left[t+2(v+w)(1-r) r^{n}+2(1-r)^{2} r^{2 n}, t+2(r u+v+w) r^{n}+\left(2+r^{2}\right) r^{2 n}\right] .
$$

Since $u>r^{n}$, we have

$$
\begin{aligned}
& t+2(u+v+w) r^{n+1}+3 r^{2 n+2}-\left(t+2 w(1-r) r^{n}+(1-r)^{2} r^{2 n}\right) \\
= & 2(r u+r v+2 r w-w) r^{n}+\left(2 r^{2}+2 r-1\right) r^{2 n} \\
\geq & 2(3 r-1) w r^{n}+2 u r^{n+1}+(2 r-1) r^{2 n} \\
> & 2(3 r-1) w r^{n}+(4 r-1) r^{2 n}>0,
\end{aligned}
$$

and

$$
\begin{aligned}
& t+2(r u+r v+w) r^{n}+\left(1+2 r^{2}\right) r^{2 n}-\left(t+2 v(1-r) r^{n}+(1-r)^{2} r^{2 n}\right) \\
= & 2(r u+2 r v-v+w) r^{n}+(r+2) r^{2 n+1} \\
\geq & 2(3 r-1) v r^{n}+(r+2) r^{2 n+1}>0,
\end{aligned}
$$


and

$$
\begin{aligned}
& t+2(r u+v+r w) r^{n}+\left(1+2 r^{2}\right) r^{2 n}-\left(t+2(v+w)(1-r) r^{n}+2(1-r)^{2} r^{2 n}\right) \\
= & 2(r u+r v+2 r w-w) r^{n}+(4 r-1) r^{2 n} \\
\geq & 2(4 r-1) w r^{n}+(4 r-1) r^{2 n}>0 .
\end{aligned}
$$

Therefore, we have

$$
g\left(I_{u, 0} \times\left(I_{v, 0} \cup I_{v, 1}\right) \times\left(I_{w, 0} \cup I_{w, 1}\right)\right)=\left[t, t+2(r u+v+w) r^{n}+\left(2+r^{2}\right) r^{2 n}\right] .
$$

It follows from condition (2.3) that

$$
t+2(r u+v+w) r^{n}+\left(2+r^{2}\right) r^{2 n} \geq t+2 u(1-r) r^{n}+(1-r)^{2} r^{2 n} .
$$

Thus, the intervals in (2.4) and (2.5) overlap and so

$$
g\left(\left(I_{u, 0} \cup I_{u, 1}\right) \times\left(I_{v, 0} \cup I_{v, 1}\right) \times\left(I_{w, 0} \cup I_{w, 1}\right)\right)=\left[t, t+2(u+v+w) r^{n}+3 r^{2 n}\right] .
$$

Note that

$$
g\left(I_{u} \times I_{v} \times I_{w}\right)=\left[t, t+2(u+v+w) r^{n}+3 r^{2 n}\right] .
$$

Therefore, we conclude that

$$
g\left(I_{u} \times I_{v} \times I_{w}\right)=g\left(\left(I_{u, 0} \cup I_{u, 1}\right) \times\left(I_{v, 0} \cup I_{v, 1}\right) \times\left(I_{w, 0} \cup I_{w, 1}\right)\right),
$$

as desired.

Lemma 2.4. Let $\alpha \geq 3$. For any $u, v, w \in L_{n}$, if

$$
2(1-r) \max \{u, v, w\}+(1-2 r) r^{n} \leq u+v+w,
$$

then

$$
g\left(I_{u} \times I_{v} \times I_{w}\right) \subseteq g\left(C_{\alpha}^{3}\right)
$$

Proof. For $k \geq n$, we define

$$
\mathcal{F}_{1, k}=\left\{I \in \mathcal{F}_{k}: I \subseteq I_{u}\right\}, \mathcal{F}_{2, k}=\left\{I \in \mathcal{F}_{k}: I \subseteq I_{v}\right\}, \mathcal{F}_{3, k}=\left\{I \in \mathcal{F}_{k}: I \subseteq I_{w}\right\},
$$

and

$$
F_{1, k}=\bigcup_{A \in \mathcal{F}_{1, k}} A, \quad F_{2, k}=\bigcup_{A \in \mathcal{F}_{2, k}} A, \quad F_{3, k}=\bigcup_{A \in \mathcal{F}_{3, k}} A .
$$

By Corollary 2.2, it suffices to show that for $k \geq n$,

$$
g\left(I_{u} \times I_{v} \times I_{w}\right) \subseteq g\left(F_{1, k} \times F_{1, k} \times F_{1, k}\right) .
$$

We now prove it by induction on $k$.

When $k=n$, we have $F_{1, n}=I_{u}, F_{2, n}=I_{v}, F_{3, n}=I_{w}$, and thus

$$
g\left(I_{u} \times I_{v} \times I_{w}\right) \subseteq g\left(F_{1, n} \times F_{2, n} \times F_{3, n}\right) .
$$

Next, assume that (2.7) is true for some $m \geq n$, i.e.,

$$
g\left(I_{u} \times I_{v} \times I_{w}\right) \subseteq g\left(F_{1, m} \times F_{2, m} \times F_{3, m}\right) .
$$

Then, taking $x \in g\left(I_{u} \times I_{v} \times I_{w}\right)$, it follows from (2.8) that there exist $u^{\prime}, v^{\prime}, w^{\prime} \in L_{m}$ such that

$$
I_{u^{\prime}} \subseteq I_{u}, I_{v^{\prime}} \subseteq I_{v}, I_{w^{\prime}} \subseteq I_{w} \text { and } x \in g\left(I_{u^{\prime}} \times I_{v^{\prime}} \times I_{w^{\prime}}\right) .
$$

Now condition (2.6) implies that $\max \{u, v, w\}>0$, and thus

$$
\max \left\{u^{\prime}, v^{\prime}, w^{\prime}\right\}>0 \text {. }
$$


Moreover, it follows from (2.6) that

$$
\begin{aligned}
(1-2 r) \max \left\{u^{\prime}, v^{\prime}, w^{\prime}\right\} & \leq(1-2 r) \max \{u, v, w\}+(1-2 r) r^{n} \\
& \leq u+v+w-\max \{u, v, w\} \\
& \leq u^{\prime}+v^{\prime}+w^{\prime}-\max \left\{u^{\prime}, v^{\prime}, w^{\prime}\right\},
\end{aligned}
$$

i.e.,

$$
2(1-r) \max \left\{u^{\prime}, v^{\prime}, w^{\prime}\right\} \leq u^{\prime}+v^{\prime}+w^{\prime} .
$$

Thus, applying Lemma 2.3, there exist $i, j, \ell \in\{0,1\}$ such that

$$
x \in g\left(I_{u^{\prime}, i} \times I_{v^{\prime}, j} \times I_{w^{\prime}, \ell}\right) .
$$

Obviously, we have $I_{u^{\prime}, i} \in \mathcal{F}_{1, m+1}, I_{v^{\prime}, j} \in \mathcal{F}_{1, m+1}$ and $I_{w^{\prime}, \ell} \in \mathcal{F}_{1, m+1}$. Therefore,

$$
x \in g\left(F_{1, m+1} \times F_{2, m+1} \times F_{3, m+1}\right) .
$$

This shows that (2.7) is true for $k=m+1$.

Corollary 2.5. For $\alpha \geq 3$,

$$
[a, b] \cup\left[2(1-r)^{2}, 3\right] \subseteq g\left(C_{\alpha}^{3}\right),
$$

where $a=2 r^{4}-4 r^{3}+3 r^{2}-2 r+1$ and $b=r^{4}-2 r^{3}+5 r^{2}-2 r+1$.

Proof. Note that

$$
\begin{aligned}
& g([0, r] \times[1-r, 1] \times[1-r, 1]) \cup g([1-r, 1] \times[1-r, 1] \times[1-r, 1]) \\
& =\left[2(1-r)^{2}, 2+r^{2}\right] \cup\left[3(1-r)^{2}, 3\right]=\left[2(1-r)^{2}, 3\right]
\end{aligned}
$$

and

$$
g\left(\left[r-r^{2}, r\right] \times\left[r-r^{2}, r\right] \times\left[1-r, 1-r+r^{2}\right]\right)=[a, b] .
$$

We claim that the intervals $g([0, r] \times[1-r, 1] \times[1-r, 1]), g([1-r, 1] \times[1-r, 1] \times[1-r, 1])$ and $g\left(\left[r-r^{2}, r\right] \times\left[r-r^{2}, r\right] \times\left[1-r, 1-r+r^{2}\right]\right)$ are all included in $g\left(C_{\alpha}^{3}\right)$. By Lemma 2.4 these just are done by checking condition (2.6). In fact, we have

$$
\begin{aligned}
& 2(1-r) \cdot(1-r)+(1-2 r) r-2(1-r)=-r<0, \\
& 2(1-r) \cdot(1-r)+(1-2 r) r-3(1-r)=-1<0,
\end{aligned}
$$

and

$$
\begin{aligned}
& 2(1-r) \cdot(1-r)+(1-2 r) r^{2}-\left(2\left(r-r^{2}\right)+(1-r)\right) \\
= & -2 r^{3}+5 r^{2}-5 r+1=-r(2 r-1)(r-2)-(3 r-1)<0 .
\end{aligned}
$$

\section{The Proof of Theorem 1.1}

For $E \subseteq \mathbb{R}$ and $t \in \mathbb{R}$, we define $t \cdot E=\{t x: x \in E\}$.

Lemma 3.1. If $E \subseteq f\left(C_{\alpha}^{4}\right)$, then $r^{2} \cdot E \subseteq f\left(C_{\alpha}^{4}\right)$. Similarly, if $E \subseteq g\left(C_{\alpha}^{3}\right)$, then $r^{2} \cdot E \subseteq g\left(C_{\alpha}^{3}\right)$.

Proof. Assume that $E \subseteq f\left(C_{\alpha}^{4}\right)$. For $x \in E$, there are $x_{1}, x_{2}, x_{3}, x_{4} \in C_{\alpha}$ such that $x=$ $x_{1}^{2}+x_{2}^{2}+x_{3}^{2}+x_{4}^{2}$. Then $r^{2} x=\left(r x_{1}\right)^{2}+\left(r x_{2}\right)^{2}+\left(r x_{3}\right)^{2}+\left(r x_{4}\right)^{2} \in f\left(C_{\alpha}^{4}\right)$. It follows that $r^{2} \cdot E \subseteq f\left(C_{\alpha}^{4}\right)$.

Similarly, the result for $g\left(C_{\alpha}^{3}\right)$ can be proved. 
Lemma 3.2. $f\left(C_{\alpha}^{4}\right)=[0,4]$ if and only if $\left(4 r^{2}, 4\right] \subseteq f\left(C_{\alpha}^{4}\right)$.

Proof. Note that

$$
0 \in f\left(C_{\alpha}^{4}\right) \text { and }(0,4]=\bigcup_{n=0}^{\infty} r^{2 n} \cdot\left(4 r^{2}, 4\right] .
$$

The sufficiency follows from Lemma 3.1.

Now we are ready to prove Theorem [1.1.

The proof of Theorem 1.1. For $1<\alpha<3$, we have $0<r<\frac{1}{3}$, which implies $4 r^{2}<(1-r)^{2}$. Thus,

$$
\left(4 r^{2},(1-r)^{2}\right) \cap f\left(C_{\alpha}^{4}\right) \subseteq\left(4 r^{2},(1-r)^{2}\right) \cap f\left(F_{1}^{4}\right)=\emptyset .
$$

Therefore, it suffices to show $f\left(C_{\alpha}^{4}\right)=[0,4]$ when $\alpha \geq 3$.

Assume that $\alpha \geq 3$. Note that $\frac{1}{3} \leq r<\frac{1}{2}$. Then we have $(1-r)^{2} \leq 4 r^{2}$. By Lemma 3.2 , it suffices to prove that

$$
\left((1-r)^{2}, 4\right] \subseteq f\left(C_{\alpha}^{4}\right)
$$

In Corollary 2.5, we have $\left[2(1-r)^{2}, 3\right] \subseteq g\left(C_{\alpha}^{3}\right)$. Thus

$$
f\left(C_{\alpha}^{4}\right) \supseteq f\left(C_{\alpha}^{3} \times\{0,1\}\right)=\left(g\left(C_{\alpha}^{3}\right)+0^{2}\right) \cup\left(g\left(C_{\alpha}^{3}\right)+1^{2}\right) \supseteq\left[2(1-r)^{2}, 4\right]
$$

Applying Corollary 2.5 and Lemma 3.1, we have

$$
g\left(C_{\alpha}^{3}\right) \supseteq\left[a r^{2 n}, b r^{2 n}\right] \cup\left[2(1-r)^{2} r^{2 n}, 3 \cdot r^{2 n}\right] \text { for } n=0,1,2, \cdots .
$$

where $a, b$ are given in Corollary 2.5. Note that for each positive integer $n$

$$
\begin{aligned}
f\left(C_{\alpha}^{4}\right) \supseteq & f\left(C_{\alpha}^{3} \times\left\{1-r, 1-r+r^{2 n}\right\}\right) \\
= & \left(g\left(C_{\alpha}^{3}\right)+(1-r)^{2}\right) \cup\left(g\left(C_{\alpha}^{3}\right)+\left(1-r+r^{2 n}\right)^{2}\right) \\
\supseteq & {\left[a r^{2 n-2}+(1-r)^{2}, b r^{2 n-2}+(1-r)^{2}\right] } \\
& \cup\left[a r^{2 n-2}+\left(1-r+r^{2 n}\right)^{2}, b r^{2 n-2}+\left(1-r+r^{2 n}\right)^{2}\right] \\
= & {\left[a r^{2 n-2}+(1-r)^{2}, b r^{2 n-2}+\left(1-r+r^{2 n}\right)^{2}\right] } \\
\supseteq & {\left[(1-r)^{2}+a r^{2 n-2},(1-r)^{2}+\left(b+2 r^{2}-2 r^{3}\right) r^{2 n-2}\right] }
\end{aligned}
$$

where the last equality and the last inclusion hold because

$$
\begin{aligned}
& b r^{2 n-2}+(1-r)^{2}-\left(a r^{2 n-2}+\left(1-r+r^{2 n}\right)^{2}\right) \\
= & (b-a) r^{2 n-2}-2(1-r) r^{2 n}-r^{4 n} \\
= & \left(4 r-r^{2}-r^{2 n}\right) r^{2 n} \geq\left(4 r-2 r^{2}\right) r^{2 n}>0,
\end{aligned}
$$

and

$$
b r^{2 n-2}+\left(1-r+r^{2 n}\right)^{2}-\left((1-r)^{2}+\left(b+2 r^{2}-2 r^{3}\right) r^{2 n-2}\right)=r^{4 n}>0 .
$$


Note that for each positive integer $n$

$$
\begin{aligned}
f\left(C_{\alpha}^{4}\right) \supseteq & f\left(C_{\alpha}^{3} \times\left\{1-r, 1-r+r^{2 n}, 1-r+r^{2 n-1}-r^{2 n}\right\}\right) \\
= & \left(g\left(C_{\alpha}^{3}\right)+(1-r)^{2}\right) \cup\left(g\left(C_{\alpha}^{3}\right)+\left(1-r+r^{2 n}\right)^{2}\right) \\
& \cup\left(g\left(C_{\alpha}^{3}\right)+\left(1-r+r^{2 n-1}-r^{2 n}\right)^{2}\right) \\
\supseteq & {\left[2(1-r)^{2} r^{2 n}+(1-r)^{2}, 3 r^{2 n}+(1-r)^{2}\right] } \\
& \cup\left[2(1-r)^{2} r^{2 n}+\left(1-r+r^{2 n}\right)^{2}, 3 r^{2 n}+\left(1-r+r^{2 n}\right)^{2}\right] \\
& \cup\left[2(1-r)^{2} r^{2 n}+\left(1-r+r^{2 n-1}-r^{2 n}\right)^{2}, 3 r^{2 n}+\left(1-r+r^{2 n-1}-r^{2 n}\right)^{2}\right] \\
= & {\left[2(1-r)^{2} r^{2 n}+(1-r)^{2}, 3 r^{2 n}+\left(1-r+r^{2 n-1}-r^{2 n}\right)^{2}\right] } \\
\supseteq & {\left[(1-r)^{2}+2(1-r)^{2} r^{2 n},(1-r)^{2}+\left(2-r+2 r^{2}\right) r^{2 n-1}\right] }
\end{aligned}
$$

where the last equality and inclusion hold because

$$
\begin{aligned}
& 3 r^{2 n}+(1-r)^{2}-\left(2(1-r)^{2} r^{2 n}+\left(1-r+r^{2 n}\right)^{2}\right) \\
= & \left(6 r-2 r^{2}-1-r^{2 n}\right) r^{2 n} \\
\geq & \left(6 r-3 r^{2}-1\right) r^{2 n}=[3(1-r) r+3 r-1] r^{2 n}>0, \\
& 3 r^{2 n}+\left(1-r+r^{2 n}\right)^{2}-\left(2(1-r)^{2} r^{2 n}+\left(1-r+r^{2 n-1}-r^{2 n}\right)^{2}\right) \\
= & -2 r^{2 n-1}+7 r^{2 n}-2 r^{2 n+2}-r^{4 n-2}+2 r^{4 n-1} \\
= & 2(3 r-1) r^{2 n-1}+\left(1-2 r^{2}\right) r^{2 n}-r^{4 n-2}+2 r^{4 n-1} \\
> & 2(3 r-1) r^{2 n-1}+r^{2 n+1}-r^{4 n-2}+2 r^{4 n-1} \\
\geq & 2(3 r-1) r^{2 n-1}-r^{4 n-2}+3 r^{4 n-1}=2(3 r-1) r^{2 n-1}+(3 r-1) r^{4 n-2} \geq 0,
\end{aligned}
$$

and

$$
3 r^{2 n}+\left(1-r+r^{2 n-1}-r^{2 n}\right)^{2}-\left((1-r)^{2}+\left(2-r+2 r^{2}\right) r^{2 n-1}\right)=\left(r^{2 n-1}-r^{2 n}\right)^{2}>0 .
$$

Note that

$$
\begin{aligned}
& a-\left(2-r+2 r^{2}\right) r=2 r^{4}-6 r^{3}+4 r^{2}-4 r+1 \\
& =(2 r-1) r^{3}-\left(5 r^{2}-4 r+1\right) r-(3 r-1)<0,
\end{aligned}
$$

and

$$
\begin{aligned}
& \left(b+2 r^{2}-2 r^{3}\right)-2(1-r)^{2}=r^{4}-4 r^{3}+5 r^{2}+2 r-1 \\
& =r^{4}+2 r^{2}(1-2 r)+(3 r-1)(r+1)>0 .
\end{aligned}
$$

Combining the above inequalities, (3.3) and (3.4) we have that for every $n \geq 1$,

$$
f\left(C_{\alpha}^{4}\right) \supseteq\left[(1-r)^{2}+2(1-r)^{2} r^{2 n},(1-r)^{2}+2(1-r)^{2} r^{2 n-2}\right] .
$$

Therefore,

$$
f\left(C_{\alpha}^{4}\right) \supseteq \bigcup_{n=1}^{\infty}\left[(1-r)^{2}\left(1+2 r^{2 n}\right),(1-r)^{2}\left(1+2 r^{2 n-2}\right)\right]=\left((1-r)^{2}, 3(1-r)^{2}\right] .
$$

By (3.2) and (3.5), we have $f\left(C_{\alpha}^{4}\right) \supseteq\left((1-r)^{2}, 3(1-r)^{2}\right] \cup\left[2(1-r)^{2}, 4\right]=\left((1-r)^{2}, 4\right]$, obtaining (3.1). 


\section{ACKNOWLEDGEMENTS}

The second author was supported by NSFC No. 11701302 and K.C. Wong Magna Fund in Ningbo University. The second author was also supported by Zhejiang Provincial Natural Science Foundation of China with No.LY20A010009. The third author was supported by NSFC No. 11671147, 11971097 and Science and Technology Commission of Shanghai Municipality (STCSM) No. 13dz2260400.

\section{REFERENCES}

[1] Athreya, Jayadev S.; Reznick, Bruce; Tyson, Jeremy T. Cantor set arithmetic. Amer. Math. Monthly 126 (2019), no. 1, 4-17.

[2] Cabrelli, Carlos A.; Hare, Kathryn E.; Molter, Ursula M. Sums of Cantor sets. Ergodic Theory Dynam. Systems 17 (1997), no. 6, 1299-1313.

[3] Jiang, Kan; Xi, Lifeng. Interiors of continuous images of the middle-third Cantor set. arXiv:1809.01880, 2018.

[4] Gu, Jiangwen; Jiang, Kan; Xi, Lifeng; Zhao, Bing. Multiplication on uniform $\lambda$-Cantor sets. arXiv:1910.08303, 2019.

[5] Mendes, Pedro; Oliveira, Fernando. On the topological structure of the arithmetic sum of two Cantor sets. Nonlinearity 7 (1994), no. 2, 329-343.

[6] Pawłowicz, Marta. Linear combinations of the classic Cantor set. Tatra Mt. Math. Publ. 56 (2013), 47-60.

[7] Randolph, J. F. Distances between points of the Cantor set. Amer. Math. Monthly 47(1940), no. 8: 549-551.

[8] Utz, W. R. The distance set for the Cantor discontinuum. Amer. Math. Monthly 58(1951),no. 6: $407-408$.

(Z. Wang) School of Mathematical Sciences, Shanghai Key Laboratory of PMMP, East China Normal University, Shanghai 200062, People's Republic of China

E-mail address: zhiqiangwzy@163.com

(K. Jiang) Department of Mathematics, Ningbo University, People's Republic of China

E-mail address: jiangkan@nbu.edu.cn

(W. Li) School of Mathematical Sciences, Shanghai Key Laboratory of PMMP, East China Normal University, Shanghai 200062, People's Republic of China

E-mail address: wxli@math.ecnu.edu.cn

(B. Zhao) Department of Mathematics, Ningbo University, People's Republic of China

E-mail address: zhaobing@nbu.edu.cn 